ACCEPTED TO APJ

Preprint typeset using LTEX style emulateapj v. 01/23/15

\title{
GIANT IMPACT: AN EFFICIENT MECHANISM FOR THE DEVOLATILIZATION OF SUPER-EARTHS
}

\author{
SHANG-FEI LIU (刘尚飞) ${ }^{1}$, YASUNORI HORI (堀安範) ${ }^{2,4}$, D.N.C. LIN ${ }^{2,5,6}$ AND ERIK ASPHAUG ${ }^{3}$ \\ ${ }^{1}$ Department of Earth and Planetary Sciences, University of California, Santa Cruz, CA 95064, USA; sliu26@ucsc.edu \\ ${ }^{2}$ Department of Astronomy and Astrophysics, University of California, Santa Cruz, CA 95064, USA; yahori@ ucsc.edu, lin@ucolick.org \\ ${ }^{3}$ School of Earth and Space Exploration, Arizona State University, Tempe, AZ 85287, USA; easphaug @ asu.edu \\ ${ }^{4}$ Astrobiology Center, National Institute of Natural Sciences and National Astronomical Observatory of Japan, Osawa 2-21-1, Mitaka, Tokyo 1818588, Japan \\ ${ }^{5}$ Kavli Institute for Astronomy \& Astrophysics, Peking University, Beijing, China \\ ${ }^{6}$ Institute for Advanced Studies, Tsinghua University and National Astronomical Observatory of China, Beijing, China \\ Accepted to ApJ
}

\begin{abstract}
Mini-Neptunes and volatile-poor super-Earths coexist on adjacent orbits in proximity to host stars such as Kepler-36 and Kepler-11. Several post-formation processes have been proposed for explaining the origin of the compositional diversity between neighboring planets: the mass loss via stellar XUV irradiation, degassing of accreted material, and in-situ accumulation of the disk gas. Close-in planets are also likely to experience giant impacts during the advanced stage of planet formation. This study examines the possibility of transforming volatile-rich super-Earths / mini-Neptunes into volatile-depleted super-Earths through giant impacts. We present the results of three-dimensional hydrodynamic simulations of giant impacts in the accretionary and disruptive regimes. Target planets are modeled with a three-layered structure composed of an iron core, silicate mantle and hydrogen/helium envelope. In the disruptive case, the giant impact can remove most of the $\mathrm{H} / \mathrm{He}$ atmosphere immediately and homogenize the refractory material in the planetary interior. In the accretionary case, the planet is able to retain more than half of the original gaseous envelope, while a compositional gradient suppresses efficient heat transfer as the planetary interior undergoes double-diffusive convection. After the giant impact, a hot and inflated planet cools and contracts slowly. The extended atmosphere enhances the mass loss via both a Parker wind induced by thermal pressure and hydrodynamic escape driven by the stellar XUV irradiation. As a result, the entire gaseous envelope is expected to be lost due to the combination of those processes in both cases. Based on our results, we propose that Kepler-36b may have been significantly devolatilized by giant impacts, while a substantial fraction of Kepler-36c's atmosphere may remain intact. Furthermore, the stochastic nature of giant impacts may account for the observed large dispersion in the mass-radius relationship of close-in super-Earths and mini-Neptunes (at least to some extent).
\end{abstract}

Subject headings: equation of state — hydrodynamics — planets and satellites: formation — planets and satellites: interiors — stars: individual (Kepler-36, Kepler-11)

\section{INTRODUCTION}

Data obtained from the Kepler mission (Borucki et al. 2010) indicate an abundant population of close-in planets with radii of $R_{\mathrm{p}}=1-4 R_{\oplus}$ (Petigura et al. 2013; Burke et al. 2014; Dressing \& Charbonneau 2015). Although the masses of only a handful of those planets have been measured with radialvelocity or transit-timing variations (TTV) observations, a large range of their mean densities $\rho_{\mathrm{p}}$ indicates that they have diverse bulk compositions (e.g. see Fig.4 in Dressing et al. 2015). These kinematic and structural properties impose constraints on theories of planetary origin.

It has been suggested that these planets may have formed through the coagulation of grains and planetesimals at their present-day location (Hansen \& Murray 2012; Chiang \& Laughlin 2013). Although all volatile grains in their natal disks are sublimated in the proximity of their central stars, embryos may have formed prolifically from refractory grains (Li et al. submitted) and acquired or retained a modest amount of hydrogen/helium atmosphere in situ with relatively low apparent mean densities (Ikoma \& Hori 2012).

Close-in planets are exposed to intense XUV (extreme UV and X-ray) irradiation from their host stars. Photoevaporation can significantly modify the structure of their atmosphere (e.g., Owen \& Wu 2013). One possible cause for the apparent dispersion in $\rho_{\mathrm{p}}$ is the retention efficiency of $\mathrm{H} / \mathrm{He}$ atmospheres among planets around host stars (Lissauer et al. 2013) with different XUV luminosities during their pre-main sequence stage. But around some host stars, high-density super-
Earths and low-density hot-Neptunes with similar $M_{\mathrm{p}}$ and $P$ (where $M_{\mathrm{p}}$ is the planetary mass and $P$ is the orbital period) coexist. Their $\rho_{\mathrm{p}}$ diversity cannot be simply attributed to the XUV flux from their common host stars.

Around a particular sub-giant star, two planets, Kepler-36b and c, were found (Carter et al. 2012) with adjacent orbits $(P=$ 13.8 and 16.2 days $)$, comparable masses $\left(M_{\mathrm{p}}=4.46 \pm 0.3 M_{\oplus}\right.$ and $\left.8.10 \pm 0.53 M_{\oplus}\right)$, and radii $\left(R_{\mathrm{p}}=1.48 \pm 0.03 R_{\oplus}\right.$ and $\left.3.67 \pm 0.05 R_{\oplus}\right)$. The inferred $\rho_{\mathrm{p}}$ of the inner super-Earth (Kepler-36b) matches that of an Earth-like composition and that of the outer mini-Neptune (Kepler-36c) is consistent with an internal composition of a thick hydrogen/helium $(\mathrm{H} / \mathrm{He})$ envelope (8.6 $\pm 1.3 \mathrm{wt} . \%$, assuming an Earth-like core) atop a rocky/iron core (Lopez \& Fortney 2013). Although a fraction of Kepler 36c's atmosphere may be due to the degassing of accreted planetesimals (Elkins-Tanton \& Seager 2008) or in-situ the accumulation of a residual disk gas onto its solid core (Ikoma \& Hori 2012). However, such a large density contrast between these two closely-spaced planets is incompatible with either of these two accretion processes.

It is possible that these two planets may once have had a similar amount of atmospheric $\mathrm{H} / \mathrm{He}$ and their compositional dichotomy could be caused by their different core masses (Lopez \& Fortney 2013). Provided that the lost mass is a small fraction of $M_{\mathrm{p}}$, their near 7:6 mean motion resonance (MMR) orbits would not be significantly affected by it (Teyssandier et al. 2015). However, Kepler 36c appears to have retained its atmosphere and Kepler $36 \mathrm{~b}$ appears to have not, albeit the for- 
mer has a smaller surface escape velocity and is expected to be more vulnerable to photoevaporation than the latter. Another system with a similar dichotomy is Kepler $11 \mathrm{~b}$ and $\mathrm{c}$ (Lissauer et al. 2011).

Another scenario is that protoplanetary embryos formed throughout the disk, including the volatile-rich cold outer regions, and converged through type I migration to their present-day locations in the proximity of their host stars (Papaloizou \& Terquem 2010; Ida et al. 2013). According to the conventional accretion scenario, protoplanetary embryos emerge through oligarchic growth and experience cohesive collisions with embryos with comparable masses (Kokubo \& Ida 1998). The energy released in such catastrophic events intensely heats both the mantle and atmosphere and may induce substantial losses of planetary atmosphere (Schlichting 2014). The giant impact hypothesis is consistent with the chaotic dynamics exhibited in the Kepler-36 system (Deck et al. 2012). Hydrodynamic and $N$-body simulations have revealed that stochastic/convergent migration can lead to close encounters between the two planets and physical collisions with other embryos (Paardekooper et al. 2013; Quillen et al. 2013).

Due to the stochastic nature of these giant impact events, structural diversity among members of closely packed, multiple short-period planet systems is the hallmark of oligarchic growth, extensive migration, and giant impacts. This diversity takes the form of different levels of shock processing, different final material inventories and planetary densities (Asphaug 2010).

In this paper we explore the possibility that structural diversity is established by giant impacts during the formation of compact multiple-planet systems. Collisions and disruptions of differentiated super-Earths with terrestrial compositions (Marcus et al. 2009) or water : rock ratios of $1: 1$ (Marcus et al. 2010) have been previously simulated with the smoothed particle hydrodynamics (SPH) scheme (Reufer et al. 2012). However, there have been no systematic simulations of giant impacts between super-Earths with $\mathrm{H} / \mathrm{He}$ atmospheres.

In $\S 2$, we outline the numerical method and the initial and boundary conditions used for these simulations. In our models, we adopt physical parameters similar to those of the $\mathrm{Ke}$ pler 36 system and scrutinize the collisional origin of the compositional diversity between Kepler-36b and c. In $\S 3$, we present the results of two sets of numerical simulations to show that energetic giant impacts can cause strong shocks and lead to the ejection of a large amount of the $\mathrm{H} / \mathrm{He}$ envelope. Shock dissipation also heats up the planetary atmosphere and interior, elevating the mass-loss rate induced by hydrodynamic escape and radiative evaporation (Hébrard et al. 2004). We summarize our results and discuss their implications in $\$ 4$.

\section{COMPUTATIONAL METHOD}

Our three-dimensional hydrodynamic simulations are based on the framework of FLASH (Fryxell et al. 2000), an Eulerian code with an adaptive-mesh refinement (AMR) capability. This finite-volume scheme has advantages over most commonly-used SPH codes in terms of capturing shock waves as well as characterizing low-density and sparse regions. Besides, advection of different fluid species in a grid cell is allowed to obey their own advection equations, which is ideally suited for mixing problems. The main purpose of this work is to investigate the general aftermath of giant impacts, so our study here is on head-on collisions.
In order to minimize the artifact of artificial diffusivity (Tasker et al. 2008), collision simulations are performed in a pair of planets' center-of-mass frame rather than in the inertial reference frame in which they move through the numerical grids with a Keplerian speed. The width of the computational domain is $1 \times 10^{13} \mathrm{~cm}$ on each side, and we choose an open boundary condition for our simulations. A multiple expansion with a large angular number $\left(L_{\max }=60\right)$ about the center of mass of the embryo pair is adopted in order to compute the self-gravity with a sufficient angular resolution.

Most hydrodynamic simulations of giant impacts ignore the presence of a central star and its tidal force. In the present context, the Hill radius of a planet, $r_{\mathrm{H}}=a(q / 3)^{1 / 3}$ (where $a$ is the semi-major axis of the planet and $q$ is the mass ratio of the planet to its parent star) is only an order of magnitude larger than its physical size. Therefore, the tidal force of the central star may have a significant effect on the planet's postimpact evolution.

The orbit of the embryo pair around the host star is determined by solving a two-body problem that treats a central star and the embryo pair as two separate point masses (Guillochon et al. 2009). Here we consider giant impacts between a target planet $M_{\mathrm{T}}$ and an Earth-mass impactor $M_{\mathrm{I}}$ at 0.1 AU separation from a $1 M_{\odot}$ star. Initially, the target and impactor are held apart from their center of mass by a few target's radii until they have relaxed into a hydrostatic equilibrium subjected to each other's tidal perturbation. They are released with their relative velocity gradually increased from rest to the values listed in Table 1. Further acceleration is determined by their mutual gravity.

Two simulations of head-on collisions in the accretionary and disruptive regimes are performed. In the accretionary model $1, M_{\mathrm{T}}=4.3 M_{\oplus}$, i.e. close to the mass of Kepler$36 \mathrm{~b}$, and the impact velocity $v_{\mathrm{imp}}=v_{\mathrm{esc}}$, where $v_{\mathrm{esc}}=$ $\sqrt{2 G\left(M_{\mathrm{T}}+M_{\mathrm{I}}\right) /\left(R_{\mathrm{T}}+R_{\mathrm{I}}\right)}$ is the two-body escape velocity. In the disruptive model $2, M_{\mathrm{T}}=10 M_{\oplus}$, i.e. the upper end of the mass spectrum that is commonly refer to super-Earths, and the impact velocity $v_{\text {imp }}=3 v_{\text {esc }}$ comparable to the local Keplerian speed. Our model 2 represents the largest scale of collisions that could happen to a close-in super-Earth with an atmosphere. Impacts onto a less massive super-Earth at the same speed lead to a more disruptive outcome.

In our models, the target is initially composed of an iron core, a silicate mantle, and an $\mathrm{H} / \mathrm{He}$ gaseous envelope (7.5 wt \%), while the impactor has the same layered structure except without an atmosphere. The mass ratio of an iron core to a silicate mantle is assumed to be $1: 2$ for all planets. We summarize the masses of each species in the targets and impactors in Table 1. The internal structure of all the target planets are resolved by a numerical mesh with $R_{\mathrm{T}} / \Delta d>100$, where $R_{\mathrm{T}}$ is the target's initial radius and $\Delta d$ is the width of the smallest grid cells.

Previously, Liu et al. (2015) have applied an early version of this scheme to investigate embryo impacts on gas giants. In those simulations, we approximated the internal structure of gas giants with a composite polytropic equation of state (EOS; Liu et al. 2013). We assume that the multi-species fluid obeys the Dalton's law, i.e. the total pressure of a mixture of gas equals the sum of the contributions of individual components. The current study focuses on collisions between smaller planets with compositions and internal structures similar to those of the Earth or Neptune. For 'terrestrial' types of planetary materials, we have incorporated the Tillotson EOS to model 
TABLE 1

MASS AND ENERGY BUDGETS FOR THE LOW AND HIGH SPEED IMPACTS. a

\begin{tabular}{cccccccccccccccccc}
\hline \hline $\begin{array}{c}v_{\text {imp }} \\
(\mathrm{km} / \mathrm{s})\end{array}$ & Iron $^{\mathrm{b}}$ & $\begin{array}{c}M_{\mathrm{T}} \\
\text { Rock }^{\mathrm{c}}\end{array}$ & $\mathrm{H} / \mathrm{He}^{\mathrm{d}}$ & \multicolumn{2}{c}{ Iron $M_{\mathrm{I}}$} & Rock & Iron & $\begin{array}{c}M \\
\text { Rock }\end{array}$ & $\mathrm{H} / \mathrm{He}$ & $U_{\mathrm{T}}$ & $E_{\mathrm{k}, \mathrm{T}}$ & $U_{\mathrm{I}}$ & $E_{\mathrm{k}, \mathrm{I}}$ & $r_{\mathrm{P}}$ & $r_{\mathrm{H}}{ }^{\mathrm{e}}$ & $r_{\mathrm{P}}$ & $r_{\mathrm{H}}$ \\
\hline 10.96 & 1.344 & 2.663 & 0.297 & 0.334 & 0.663 & 1.676 & 3.315 & 0.208 & 4.746 & 0.441 & 0.516 & 2.092 & 9.176 & 9.442 & 0.186 & 0.215 \\
53.06 & 3.115 & 6.129 & 0.746 & 0.334 & 0.662 & 3.449 & 6.289 & 0.151 & 21.890 & 4.529 & 0.407 & 72.338 & 31.039 & 32.810 & 0.240 & 0.311 \\
\hline
\end{tabular}

${ }^{\text {a }}$ Integral quantities $M, U$ and $E_{\mathrm{k}}$ correspond to mass, internal energy and kinetic energy, respectively. Subscript $\mathrm{T}$ and I denote the target and the impactor. Integral quantities without additional subscripts are measured at the end point of our simulations. Mass data are in units of Earth mass and energy data are in units of $10^{39}$ erg.

$\mathrm{b}, \mathrm{c}, \mathrm{d}$ Mass of species iron, rock and $\mathrm{H} / \mathrm{He}$, respectively.

e The integral quantity is measured within the original radius of the target (cf. Figure 2 and 7 ).

${ }^{\mathrm{f}}$ The integral quantity is measured within the Hill radius $\left(2.62 \times 10^{10}\right.$ and $3.21 \times 10^{10} \mathrm{~cm}$, respectively).
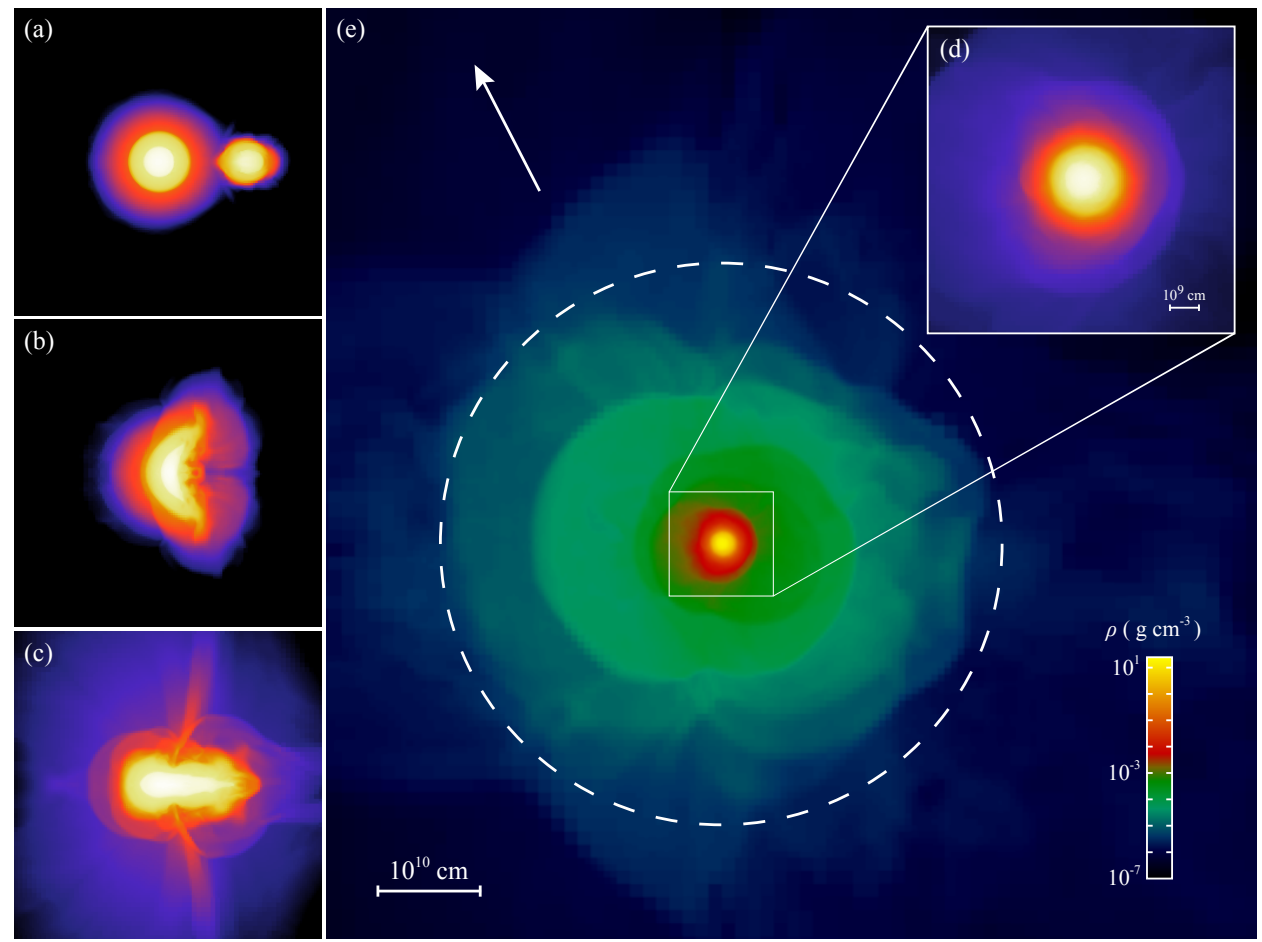

FIG. 1.- Snapshots of the giant impact simulation between a $4.3 M_{\oplus}$ super-Earth and an Earth-mass planet at the escape velocity $v_{\text {esc }}$. The density distribution across slices in the planets' orbital plane with density range $\left[10^{-4}, 2.5 \times 10^{1}\right] \mathrm{g} \mathrm{cm}^{-3}$ (represented by colors ranging from purple to white) before the impact (panel a), immediately after (b), at 1.56 hours (c), and at 18 hours after the impact (d). Panel e shows an enlarged view of the panel d. With a radius $2.62 \times 10^{10} \mathrm{~cm}$, the dashed circle overplotted on the planet indicates its Hill sphere at a distance of $0.1 \mathrm{AU}$ from a solar-mass star. The parent star is in the direction of the white arrow.

iron cores and silicate mantles (see Appendix I of Melosh 1989). We additionally model an $\mathrm{H} / \mathrm{He}$ atmosphere (70 wt. \% $\mathrm{H}_{2}$ and 30 wt. $\left.\% \mathrm{He}\right)$ assuming a polytropic EOS $\left(P \propto \rho^{\gamma}\right)$ with an adiabatic index of $\gamma=5 / 3$. This simplified assumption can be validated for temperatures below the excitation temperature of the $\mathrm{H}_{2}$ rotation band, $170 \mathrm{~K}$, and above $\mathrm{H}_{2}$ dissociation temperature $(\sim 2000 \mathrm{~K})$ but below that for the $\mathrm{H}$ and $\mathrm{He}$ atoms to be fully ionized. For the intermediate regime, our treatment underestimates the compressibility and heat capacity of hydrogen, which may exaggerate an impact-driven atmospheric escape. However, the atmosphere of the target is generally not sufficiently massive to stall the impactor and to prevent the dissipation of its kinetic energy near the target's core. We find that the computed turbulent mixing of material and the mass loss rate of the atmosphere are relatively insensitive to the specific $\mathrm{H} / \mathrm{He}$ EOS model.

Giant impacts are violent and impulsive events. A new quasi-hydrostatic equilibrium within the planet's Hill's radius is generally established within a dynamical timescale (within a day). During the transitory phase, we can neglect the effect of stellar heating on the inflated atmosphere (see $\$ 4$ ). After the impact, the efficiency of insolation due to stellar irradiation on the planet's surface and the efficiency of heat transfer in its interior determine the mass-loss rate from the inflated planet (Owen \& Wu 2015). Here we must also consider that the giant impact has left behind a massive debris torus that can remain opaque for some time.

Part of the analysis and visualization presented in Section 3 is generated using the YT package (Turk et al. 2011) and the VisIt software (Childs et al. 2012).

\section{RESULTS}

\subsection{Low-speed model 1}

The compositional structure of a super-Earth after a lowspeed collision with an Earth-mass impactor is shown in Figure 1. The initial mass of the target is $4.3 M_{\oplus}$. The impactor's approaching speed is the escape velocity of the targetimpactor system. Panels (a) - (c) show snapshots of density 


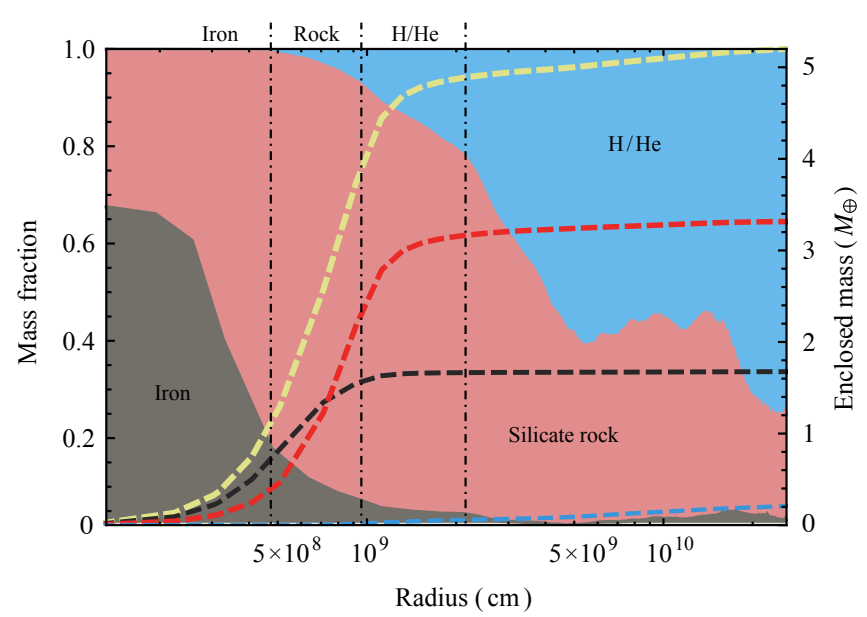

FIG. 2.- Each shaded area illustrates the mass fraction of each species as a function of radius at 18 hours after a low-speed giant impact. The grey, light coral, and blue regions represent iron, silicate rock and $\mathrm{H} / \mathrm{He}$ gas, respectively. Three vertical dot-dashed lines indicate the initial compositional boundaries inside the target that has the three-layered structure. The dashed lines from top to bottom show the cumulative mass (yellow), the enclosed mass of silicate rock (red), iron (black), and $\mathrm{H} / \mathrm{He}$ (blue) as a function of radius in units of Earth mass.

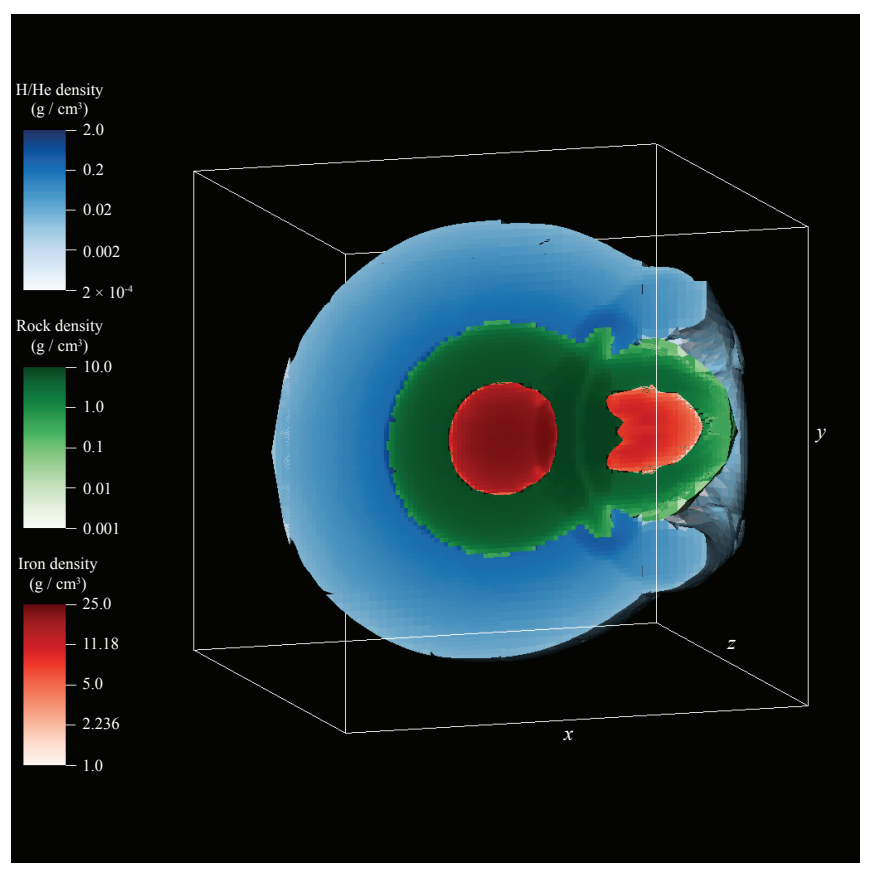

FIG. 3.- Cutaway view showing a snapshot after the contact between the rocky layers of the two planets and before the merger of their iron cores. The blue, green and red colors represent partial densities of $\mathrm{H} / \mathrm{He}$, rock and iron species, whichever is the dominant species of a grid cell. For the most part of the target, the total density of a grid cell is close to the partial density of the dominant species because mixing is not severe. However, a small fraction of $\mathrm{H} / \mathrm{He}$ (less than $5 \mathrm{wt}$. \%) being mixed with rock is responsible for the formation of a low-density arced structure between compressed rocky layers. The cubic box has a width of $4 \times 10^{9} \mathrm{~cm}$.

contours before, during, and at $1.56 \mathrm{hr}$ after the impact. The snapshots in panels (d) and (e) are taken at 18 hours after the impact. At this time, the central region of the post-impact target is mostly dynamically relaxed (see panel (d)), while the hot atmosphere extends well beyond the Hill radius and continues to lose its mass via Roche lobe overflow (see also §4).

Since the total orbital energy of the target-impactor system is nearly zero, this impact has an accretion efficiency close to but not quite one. The target gains mass after the impact (Table 1) but not all of it. The excess kinetic energy injected by the projectile drives an outflow as the planet approaches a hydrostatic equilibrium. As a result, the impact is not a perfect merger. The total mass loss (i.e. the mass that is failed to be accreted by the target) at 18 hours after the impact is $\sim 0.1 M_{\oplus}$. This value is expected to increase slightly due to the slowly decaying oscillations in the tenuous outer part of the atmosphere over a prolonged period of time. Accretion efficiency in low-velocity collisions can be substantially lower than it is for the head-on cases considered here, because off-axis giant impacts can attempt to accrete too much angular momentum for a single planet or planet-satellite system to sustain. The so-called hit-and-run regime is relevant at velocities intermediate to those considered here (Asphaug 2010).

The gravitationally unbound mass, $\sim 0.1 M_{\oplus}$, is mostly composed of $\mathrm{H} / \mathrm{He}$ gas plus a minute contribution from iron and silicate material (see Table 1). The impact leads to the immediate ejection of nearly one-third of the target's initial $\mathrm{H} / \mathrm{He}$ atmosphere. Most of the impactor's mass is added to that of the target. Consequently, the volatile fraction of the target is significantly reduced.

The enclosed mass as a function of radius inside the Hill sphere is plotted with a yellow dashed line in Figure 2. Most of the planetary mass is confined within its original radius, while the tenuous atmosphere spreads out by an order of magnitude, filling up the entire Hill sphere (see panel (e) of Figure 1). The grey, pink, and blue regions in Figure 2 illustrate iron, rock and $\mathrm{H} / \mathrm{He}$ mass fractions as functions of planet's radius. The masses of the three components enclosed within a given spherical radius are overplotted with red (rock), black (iron), and blue $(\mathrm{H} / \mathrm{He})$ dashed lines, respectively.

When the intruding planet enters the target's envelope, a velocity shear is present at the interface between two species which triggers Kelvin-Helmholtz (K-H) instabilities. On the other hand, during the propagation of the impact-induced shock wave through the target, a denser fluid is accelerated by a less dense one at their interface and Rayleigh-Taylor (R$\mathrm{T}$ ) instabilities start to develop. However, since the impactor merges with the target in less than half an hour, only smallscale instabilities arise during such a short time span (see, e.g. Agertz et al. 2007), which can only be resolved with an extremely high resolution. Figure 3 shows a cutaway view of a snapshot when the impactor is about to merge with the target. We plot the partial density, i.e. the density times the mass fraction of the dominant species of a grid cell, to illustrate the interior structure. We note that only a little mixing occurs when the target's gaseous envelope gets crushed to its rocky layer, reducing the density of rocky material in a narrow region between the target and the impactor (see Figure 3). As during the short impact phase, both $\mathrm{K}-\mathrm{H}$ and $\mathrm{R}-\mathrm{T}$ instabilities are unable to cause large scale mixing, we identify that the impact-driven turbulence is responsible for the global mixing observed during the late stages (see Figure 4). Because a large amount of kinetic energy is released upon the coalescence of the target and the impactor, fluid motions become turbulent and a complex mixture of K-H and R-T instabilities can be triggered. The turbulent mixing, as well as hydrodynamic instabilities, destroy the original layered interior structure of the target - causing radial mixing between iron and rock near the center, and inducing a fraction of rock (and a much smaller fraction of iron) to diffuse into the $\mathrm{H} / \mathrm{He}$ atmosphere (see Figure 4). Nevertheless, figure 2 shows that more than two-thirds of the mass at the center still consists of iron species. Besides, 

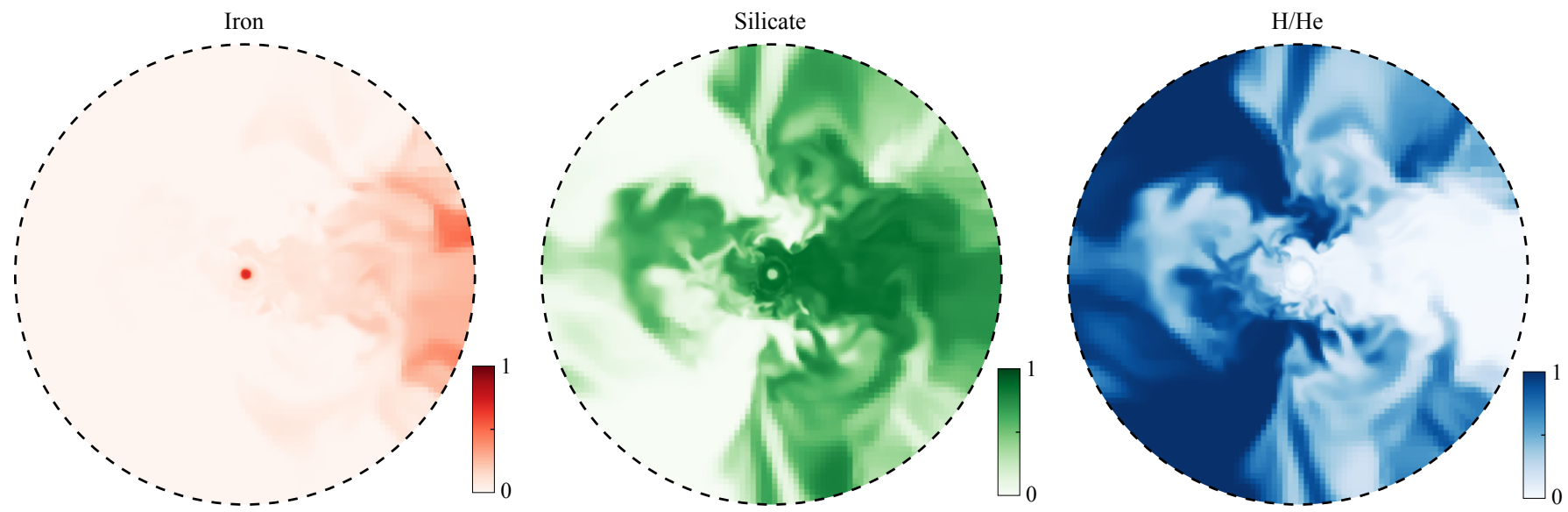

FIG. 4.- Mass fraction slices of each species through the orbital plane at $18 \mathrm{hr}$ after the low-speed impact. A significant degree of mixing is established due to the turbulent mixing as well as hydrodynamic instabilities during the post-impact expansion phase. Dashed circles represent the the Hill sphere with a radius of $2.62 \times 10^{10} \mathrm{~cm}$

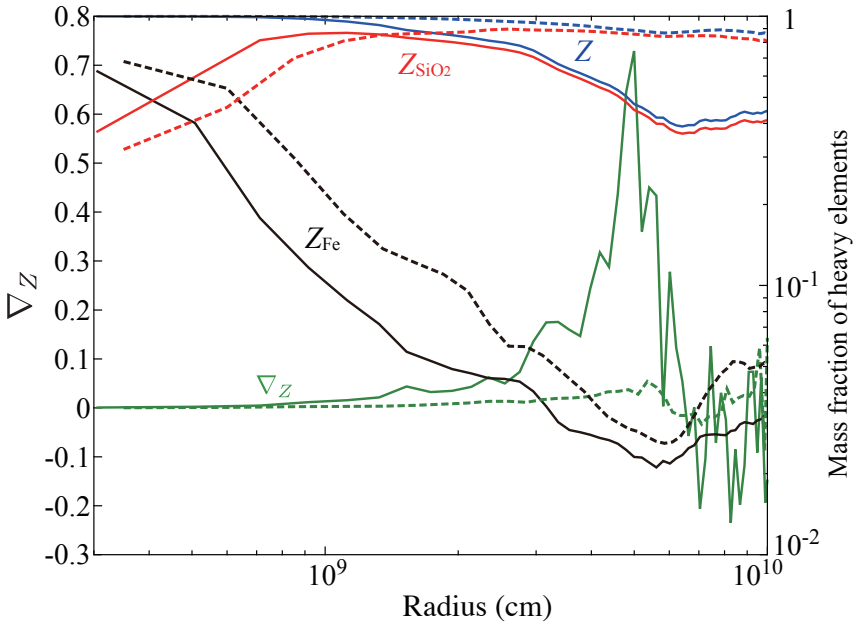

FIG. 5.- Compositional gradients inside the target planets after head-on collisions. The low-speed and high-speed impacts are represented by solid and dashed lines, respectively. Mass fractions of iron (black), rocky material (red), and heavy elements (blue) as functions of the radius are shown with labels on the right. The compositional gradient $\nabla_{Z}$ (green) increases with radius in the low-speed case. The small value of $\nabla_{Z}$ at radii indicates that the elements are well-mixed and homogenized. Fluctuation in $\nabla_{Z}$ reflects that the planet has not yet reached a relaxed state.

the iron mass fraction falls off rapidly with the radius, indicating that the planetary core survives from the impact and its mass grows in a coalescent manner.

In contrast, the rocky material is dredged up to the top of the atmosphere due to the turbulent motions driven by the impact. As shown in Figure 5, we note that the target's interior, i.e. within $6 \times 10^{9} \mathrm{~cm}$, has a steep and positive compositional gradient, $\nabla_{Z}=\mathrm{d} \log Z / \mathrm{d} \log P$, which serves to stabilize thermal convection in the mantle. At least right after the giant impacts, a head-on collision develops a hot and inhomogeneous interior. Heat transport deep inside a post-impact planet may be suppressed by the effect of double diffusion convection (e.g. Rosenblum et al. 2011; Leconte \& Chabrier 2012). Such an inefficient heat transfer mechanism would also prolong the presence of a magma ocean and delay the differentiation of iron material from a silicate-rich mantle. Off-axis collisions would lead to more efficient mixing, but also a less efficient kinetic energy contribution.

We summarize the energy budget in Table 1 . Note that the planet's internal energy is almost doubled after the impact and it is larger than the sum of the internal and kinetic energies of the target and the impactor prior to the impact. This difference arises because the collision is accretionary, i.e. a large amount of the impactor's mass is delivered to the interior of the target. As a result, not only most of the kinetic energy but also some portion of the gravitational potential energy is converted into the post-impact internal energy. Therefore, one would expect that the temperature of the planet's interior to increase substantially after the impact.

\subsection{High-speed model 2}

The consequences of a high-speed impact is similar to that of the low-speed counterpart in terms of shock propagation throughout the target after the impact, mass loss via Roche lobe overflow, and gradually decaying oscillations inside the planet (Figure 6). Despite those general similarities, the highspeed impact differs from the low-speed one in three respects.

First, the high-speed impact is erosive. The mass of the target planet at $21.5 \mathrm{hr}$ after the impact is about $9.9 M_{\oplus}$, i.e., $0.1 M_{\oplus}$ is stripped off from the target by the impactor. Thus, the total mass loss is $\sim 1.1 M_{\oplus}$. Besides, a significant amount of mass is levitated at the top of the atmosphere (see Figure 7). A breakdown of the mass budget in the Hill sphere (i.e., $3.21 \times 10^{10} \mathrm{~cm}$ in this case) shows that the impactor successfully deposits all of its iron material into the target, while most of the impactor's rocky material is ejected (see also Table 1). And the target retains about $1 / 5$ of its original atmosphere after such an energetic impact.

Second, silicate rock material become the dominant species in the planet's atmosphere (Figure 7 and 8). As a result, the compositional gradient is nearly zero out to the Hill's radius, indicating a homogeneous distribution of the heavy material inside the bulk of the target (see Figure 5). In contrast to the low-velocity model, model 1 , the negligible compositional gradient cannot suppress convection in the interior of the target planet. Nevertheless, iron material remains concentrated near the center of the planet as in the case of the low-speed model, model 1.

Third, the planet becomes less bound gravitationally. The mass contained within the target's original size is much smaller (see Figure 7). In terms of the energy budget, most of the kinetic energy is carried away by the unbound mass and only a fraction of it is directly converted to the planet's 


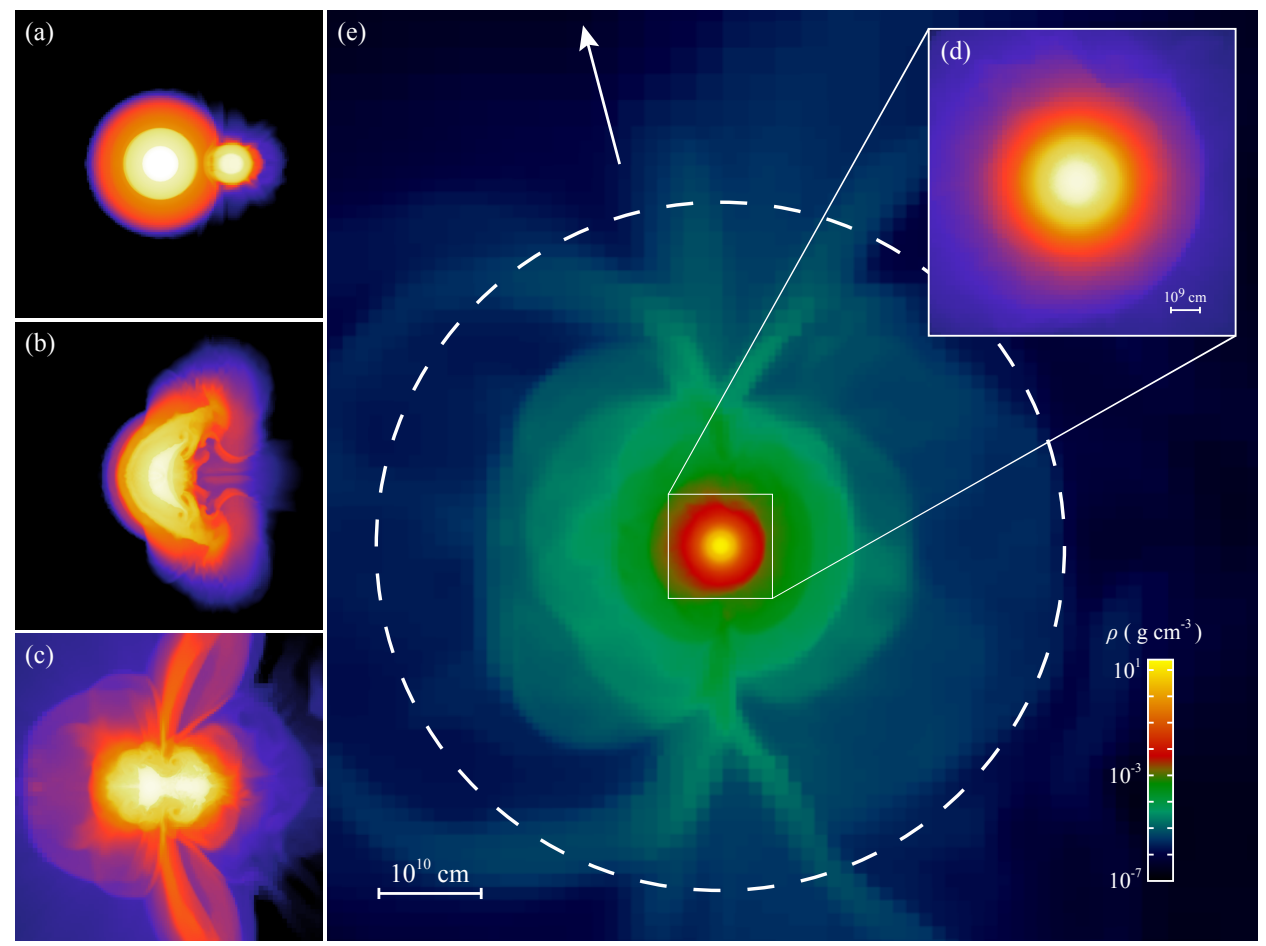

FIG. 6. - Snapshots of the giant impact simulation between a $10 M_{\oplus}$ super-Earth and an Earth-mass planet at $3 v_{\text {esc }}$. The colormaps and symbols are same as in Figure 1. Panels (a)-(d) are snapshots taken at the start of the impact, at 15 minutes, at $1.5 \mathrm{hr}$ and at $21.5 \mathrm{hr}$ after the impact, respectively. Panel (e) shows an enlarged view of the panel (d). The Hill sphere illustrated by the dashed circle has a radius of $3.21 \times 10^{10} \mathrm{~cm}$.

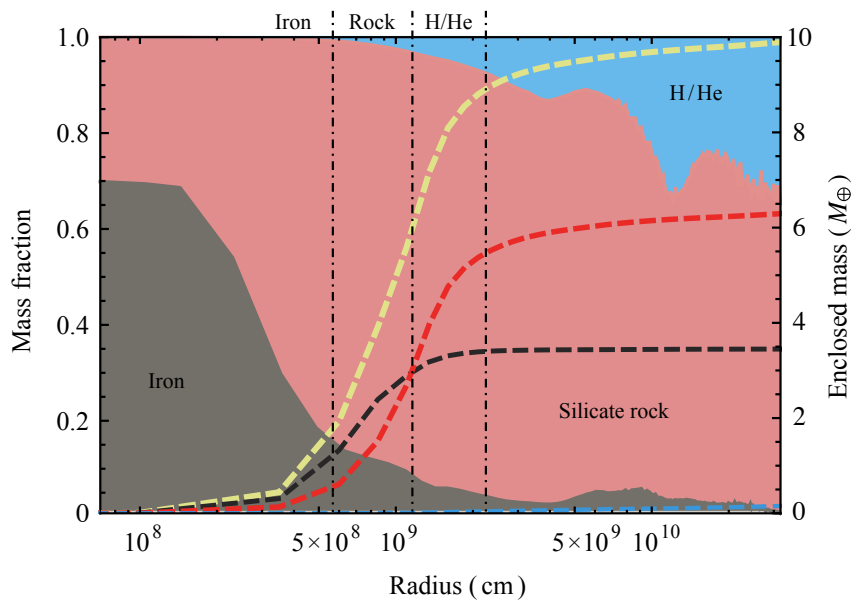

FIG. 7.- Mass fraction area plot and enclosed mass line plot at 21.5 hours after the high-speed impact. The symbols have the same meanings as in Figure 2 .

internal energy. However, the gravitational potential energy released by the sedimentation of rock material in the atmosphere becomes an extra heat source for the target interior. Assuming that silicates in the $\mathrm{H} / \mathrm{He}$ atmosphere establish a local thermal equilibrium with the turbulent gas, they condense into grains in cooler outer regions with $r \gtrsim 10^{10} \mathrm{~cm}$. These silicate condensates grow through collisional coagulation (e.g. Podolak 2003). Relatively large silicate grains are expected to settle toward the planetary surface and sublimate along the way.

\section{DISCUSSION AND CONCLUSIONS}

The results presented in the previous sections show the planet's structure immediately after head-on giant impacts. Models 1 and 2 illustrate the possibility of diverse instanta- neous outcomes depending on energy of collision, one at low velocity and the other at approximately the local Keplerian velocity, three times the planets' escape velocity. Whereas the initial gaseous envelope of a target super-Earth is mostly retained during the low-velocity impact, it is severely depleted shortly after the high-velocity impact. In this context, we suggest that giant impacts of varying energy can effectively devolatilize super-Earths/mini-Neptunes, and can diversify the compositions and interior structures of these mid-sized extrasolar planets, perhaps as occurred in our terrestrial system (see, e.g. Asphaug 2014).

The interior and atmosphere of a post-impact planet can achieve a thermal equilibrium state after a long-term evolution, which is difficult and not cost-effective to study by performing hydrodynamic simulations. Alternatively, the radiative cooling timescale $\left(\tau_{\text {rad }}\right)$ can be estimated from the rate of temperature change in an atmospheric layer via the outgoing infrared radiation: $\tau_{\text {rad }} \sim P C_{\mathrm{p}} /\left(4 g \sigma T^{3}\right)$, where $P$ and $T$ are the pressure and temperature near the photosphere obtained from the end state of hydrodynamic simulations, $g$ is the surface gravity, and $C_{\mathrm{p}}$ is the specific heat capacity at a constant pressure, and $\sigma$ is the Stefan-Boltzmann constant. Under the intense radiation from a $1 M_{\odot}$ central star, an isothermal layer develops in the planet's residual upper atmosphere.

At the current location of Kepler $36 \mathrm{~b}$ and c, the equilibrium temperature due to stellar irradiation is $\sim 900 \mathrm{~K}$. Adopting EOS of an ideal gas for $\mathrm{H} / \mathrm{He}$ and the gas opacity of Freedman et al. (2008), we find that the H/He gas within the Hill sphere is optically thick. At the photosphere, we used $P \sim 2 g / 3 \kappa$ and $\kappa$ is the opacity to estimate $\tau_{\text {rad }}$ to be larger than several days. This estimate justifies, a posteriori the neglect of stellar irradiation in our impact simulations (see \$2). Since this cooling time scale is much longer than the dynamical time scale, we can also assume that after a giant impact, a heated planet 

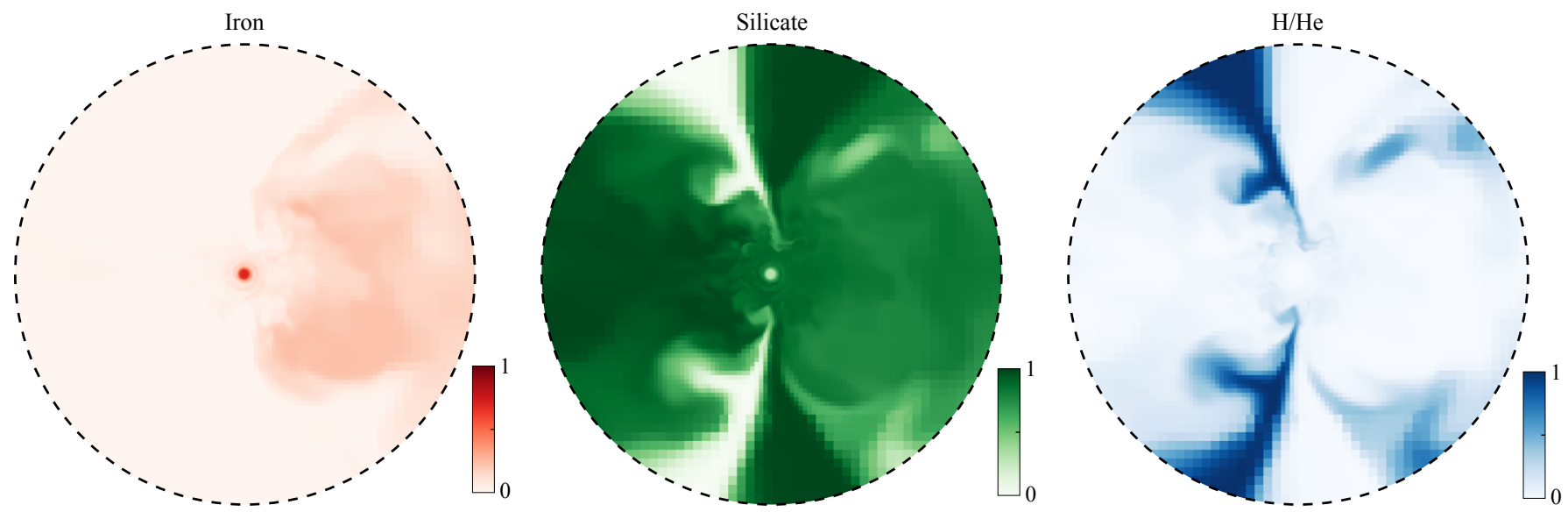

FIG. 8.- The mass fraction slices of each species through the orbital plane at 21.5 hours after the high-speed impact. Mixing becomes less efficient because most of the $\mathrm{H} / \mathrm{He}$ envelope has been blown away by the impactor. Dashed circles represent the Hill sphere with a radius of $3.21 \times 10^{10} \mathrm{~cm}$.

evolves adiabatically into a hydrostatic equilibrium.

During the post-impact phase, the planet's atmosphere and interior contract as the planet cools. Above the photosphere, a highly-irradiated planet has an isothermal layer. We consider that an inflated planet after an impact radiates away heat from the photosphere. Owen \& Wu (2015) estimated the KelvinHelmholtz timescale of a planet $\left(3,5\right.$, and $\left.10 M_{\oplus}\right)$ with the equilibrium temperature of $900 \mathrm{~K}$ as a function of planetary radius in unit of the Bondi radius and envelope mass fraction based on a stellar evolution code, i.e., MESA (Paxton et al. 2011). We applied their results of 5 and $10 M_{\oplus}$ shown in Figure 2 in Owen \& Wu (2015) to our targets after giant impacts $\left(\sim 4.991 M_{\oplus}\right.$ and $9.738 M_{\oplus}$ with the equilibrium temperature of $\sim 900 \mathrm{~K})$.

Following results of Owen \& Wu (2015), we estimate the timescale of the Kelvin-Helmholtz contraction to be $\sim 1 \mathrm{Myr}$ for the low-speed case and $<10 \mathrm{kyr}$ for the high-speed one. A protracted state of a hot and inflated atmosphere enhances its mass loss via a Parker wind. Based on Eq.(16) in Owen \& $\mathrm{Wu}(2015)$, we estimate that the amount of mass loss for postimpact planets via a Parker wind for the two cases would be $\sim 88 \%$ and $\sim 80 \%$ of its envelope mass right after the impact.

As the planet cools down, silicate material sediments from its atmosphere and contracts within its interior. The release of gravitational energy provides a source of internal heat which prolongs the inflationary state of the planet's atmosphere and enhances mass loss via a Parker wind. The extended planetary atmosphere also intercepts a greater fraction of the stellar Xray and UV irradiation and increases the rate of mass loss through photoevaporation.

We estimate the mass loss rate of an extended atmosphere via stellar XUV irradiation after giant impacts. Adopting analytical descriptions of a XUV energy flux from solar-type stars (Ribas et al. 2005), the incident XUV luminosity at the Hill radius is $1.0 \times 10^{-7} L_{\odot}$ and $1.5 \times 10^{-7} L_{\odot}$ in the low-speed and high-speed model, respectively. Given that the conversion efficiency from XUV photons to the kinetic energy of bulk atmospheric outflow is $10 \%$ as a fiducial value (Yelle 2004), the mass loss rate from the Roche lobe via stellar XUV irradiation right after giant impacts is estimated to be $\sim 3 M_{\oplus} \mathrm{Myr}^{-1}$ for the former and $\sim 2 M_{\oplus} \mathrm{Myr}^{-1}$ for the latter. If we consider a typical decay timescale of a XUV flux for Sun-like stars is $\sim 0.1 \mathrm{Gyr}$, the planet in the low-speed model may lose the entire envelope because its atmosphere shrinks in a few Myr. On the other hands, the planet in the high-speed model can contract more quickly $(<10 \mathrm{kyr})$, but it is unlikely for its tenuous post-impact atmosphere to survive a subsequent mass loss via stellar irradiation, either. Note that the stellar tidal field is crucial in this context because it can continuously remove planetary outer atmosphere that is beyond the Hill sphere. For giant impacts happen further away from the host star, tidal stripping becomes inefficient. And the outcome of a giant impact is determined by the mass ratio, impact speed and impact angle (Asphaug 2010; Leinhardt \& Stewart 2012; Stewart \& Leinhardt 2012).

To summarize, severe giant impacts can significantly devolatilize close-in super-Earths and mini-Neptunes. The compositional dichotomy between Kepler-36 b and c can be explained by their distinct impact histories along with the formation of the closely packed system, i.e. Kepler-36b experienced substantial giant impacts, and in the meanwhile Kepler$36 \mathrm{c}$ survived from being heavily bombarded. In addition, we speculate that giant impacts may have been imprinted in the large dispersion in the mass-radius relationship of close-in sub-Neptune-sized planets, as giant impacts occur stochastically and can diversify planetary interior and atmosphere otherwise well constrained.

In our solar system, there may be evidence for a similar though more subtle dichotomy. Despite their similar masses, radii, and compositions, thermal evolution models of Uranus and Neptune suggest that right after their formation, Neptune may have been relatively luminous but Uranus relatively faint (e.g. Hubbard \& Macfarlane 1980; Fortney et al. 2011). Stevenson (1986) suggested that this dichotomy may be accounted for if a violent head-on collision yielded a hot and homogeneous interior of Neptune, whereas an oblique collision caused a tilted Uranus with a stably-stratified interior. The results presented here imply that the former scenario for Neptune may be possible.

We thank Jonathan Fortney, Pascale Garaud, James Guillochon, Don Korycansky, and Xiaojia Zhang for fruitful discussions. We also thank an anonymous referee for helpful suggestions to improve the clarity of this manuscript. S.-F.L. and E.A. are sponsored by the NASA grant NNX13AR66G. Y.H. is supported by a Grant-in-Aid for JSPS Fellows (No.25000465) and a Grant-in-Aid for Scientific Research on Innovative Areas (No. 26103711) from MEXT of Japan. Sup- 
port from a UC/Lab Fee grant, an IGPPS grant, and the NSF grant 1211394 are also acknowledged. Numerical computations were carried out on the Laohu cluster at NAOC, on Cray XC30 at NAOJ, and on the Hyades cluster at UCSC. The FLASH code used in this work was in part developed by the
DOE NNSA-ASC OASCR Flash Center at the University of Chicago. VisIt is supported by the Department of Energy with funding from the Advanced Simulation and Computing Program and the Scientific Discovery through Advanced Computing Program.

\section{REFERENCES}

Agertz, O., Moore, B., Stadel, J., et al. 2007, MNRAS, 380, 963 Asphaug. E. 2010, Chemie der Erde - Geochemistry, 70, 199

-. 2014, Annual Review of Earth and Planetary Sciences, 42, 551

Borucki, W. J., Koch, D., Basri, G., et al. 2010, Science, 327, 977

Burke, C. J., Bryson, S. T., Mullally, F., et al. 2014, ApJS, 210, 19

Carter, J. A., Agol, E., Chaplin, W. J., et al. 2012, Science, 337, 556

Chiang, E., \& Laughlin, G. 2013, MNRAS, 431, 3444

Childs, H., Brugger, E., Whitlock, B., et al. 2012, in High Performance Visualization-Enabling Extreme-Scale Scientific Insight, 357

Deck, K. M., Deck, K. M., Holman, M. J., et al. 2012, ApJ, 755, L21

Dressing, C. D., \& Charbonneau, D. 2015, ArXiv e-prints, 1501.01623

Dressing, C. D., Charbonneau, D., Dumusque, X., et al. 2015, ApJ, 800, 135

Elkins-Tanton, L. T., \& Seager, S. 2008, ApJ, 685, 1237

Fortney, J. J., Ikoma, M., Nettelmann, N., et al. 2011, ApJ, 729, 32

Freedman, R. S., Marley, M. S., \& Lodders, K. 2008, ApJS, 174, 504

Fryxell, B., Olson, K., Ricker, P., et al. 2000, ApJS, 131, 273

Guillochon, J., Ramirez-Ruiz, E., Rosswog, S., \& Kasen, D. 2009, ApJ, 705, 844

Hansen, B. M. S., \& Murray, N. 2012, ApJ, 751, 158

Hébrard, G., Lecavelier Des Étangs, A., Vidal-Madjar, A., Désert, J. M., \& Ferlet, R. 2004, Extrasolar Planets: Today and Tomorrow, 321, 203

Hubbard, W. B., \& Macfarlane, J. J. 1980, Journal of Geophysical Research, 85,225

Ida, S., Lin, D. N. C., \& Nagasawa, M. 2013, ApJ, 775, 42

Ikoma, M., \& Hori, Y. 2012, ApJ, 753, 66

Kokubo, E., \& Ida, S. 1998, Icarus, 131, 17

Leconte, J., \& Chabrier, G. 2012, A\&A 540, 20

Leinhardt, Z. M., \& Stewart, S. T. 2012, ApJ, 745, 79

Li, R., Zhang, Y., Lin, D., \& Dong, B. submitted

Lissauer, J. J., Fabrycky, D. C., Ford, E. B., et al. 2011, Nature, 470, 53

Lissauer, J. J., Jontof-Hutter, D., Rowe, J. F., et al. 2013, ApJ, 770, 13

Liu, S.-F., Agnor, C. B., Lin, D. N. C. \& Li, S.-L. 2015, MNRAS, 446, 1685

Liu, S.-F., Guillochon, J., Lin, D. N. C., \& Ramirez-Ruiz, E. 2013, ApJ, 762, 37
Lopez, E., \& Fortney, J. J. 2013, ApJ, 776, 2

Marcus, R. A., Sasselov, D., Stewart, S. T., \& Hernquist, L. 2010, ApJ, 719, L45

Marcus, R. A., Stewart, S. T., Sasselov, D., \& Hernquist, L. 2009, ApJ, 700, L118

Melosh, H. J. 1989, Impact cratering: A geologic process (Research supported by NASA. New York, Oxford University Press (Oxford Monographs on Geology and Geophysics, No. 11), 1989, 253 p.) Owen, J. E., \& Wu, Y. 2013, ApJ, 775, 105

-. 2015, ArXiv e-prints, 1506.02049

Paardekooper, S.-J., Rein, H. \& Kley, W. 2013, MNRAS, 434, 3018

Papaloizou, J. C. B., \& Terquem, C. 2010, MNRAS, 405, 573

Paxton, B., Bildsten, L., Dotter, A., Herwig, F., Lesaffre, P., \& Timmes, F. 2011, ApJS, 192, 3

Petigura, E. A., Marcy, G. W., \& Howard, A. W. 2013, ApJ, 770, 69

Podolak, M. 2003, Icarus, 165, 428

Quillen, A. C., Bodman, E., \& Moore, A. 2013, MNRAS, 435, 2256

Reufer, A., Meier, M. M. M., Benz, W., \& Wieler, R. 2012, Icarus, 221, 296

Ribas, I., Guinan, E. F., Güdel, M., \& Audard, M. 2005, ApJ, 622, 680

Rosenblum, E., Garaud, P., Traxler, A., \& Stellmach, S. 2011, ApJ, 731, 66 Schlichting, H. E. 2014, ApJ, 795, L15

Stevenson, D. J. 1986, LUNAR AND PLANETARY SCIENCE XVII, 17, 1011

Stewart, S. T., \& Leinhardt, Z. M. 2012, ApJ, 751, 32

Tasker, E. J., Brunino, R., Mitchell, N. L., et al. 2008, MNRAS, 390, 1267

Teyssandier, J., Owen, J. E., Adams, F. C., \& Quillen, A. C. 2015, MNRAS, 452,1743

Turk, M. J., Smith, B. D., Oishi, J. S., et al. 2011, ApJS, 192, 9

Yelle, R. V. 2004, Icarus, 170, 167 